56th Annual American Power Conference "Generation-Fuel Options \& Equipment" Chicago, IL - April 25-27, 1994

\section{Flywheel Energy Storage using Superconducting Magnetic Rearings}

\author{
Robert G. Abboud \\ Commonwealth Research Corporation \\ Chicago, Illinois \\ Kenneth Uherka \\ John Hull \\ Tom Mulcahy \\ Argonne National Laboratory
}

\section{Introduction}

Storage of electrical energy on a utility scale is currently not practicable for most utiliiies, preventing the full utilization of existing base-load capacity. A potential solution to this problem is Flywheel Energy Storage (FES), made possible by technological developments in high-tempurature superconducting materials. Commonwealth Research Corporation (CRC), the research arm of Commonwealth Edison Company, and Argonne National Laboratory are implementing a demonstration project to advance the state of the art in high temperature superconductor (HTS) bearing performance and the overall demonstration of efficient Flywheel Energy Storage.

Currently, electricity must be used simultaneously with its generation as electrical energy storage is not available for most utilities. Existing storage methods either are dependent on special geography, are too expensive, or are too inefficient. Without energy storage, electric utilities, such as Commonwealth Edison Company, are forced to cycle base load power plants to meet load swings in hourly customer demand. Demand can change by as much as $30 \%$ over a 12 -hour period and result in significant costs to utilities as power plant output is adjusted to meet these changes. HTS FES systems can reduce demand-based power plant cycling by storing unused nighttime capacity until it is needed to meet daytime demand.

New HTS technologies offer the possibility of constructing high-speed, near-frictionless, passive bearing assemblies. These bearings are highly stable

\section{ANR/ET'CP-82361 \\ Conf-940445--2}

and have thousands of times less friction than the best roller bearings. This presents new storage possibilities when combined with technological advances in highstrength composite materials and advanced power electronics. By incorporating HTS bearings with highstrength composite materials for ultra high-speedflywheels with new-technology motor/generators, energy can be stored and released with very low losses.

The purpose of the Commonwealth Edison/Argonne FES project is to demonstrate the technical feasibility, utility benefits, and near-term economic practicality of FES systems that use HTS magnetic bearings. The curreni near-term deliverable of the program is fabrication and ti sting of a prciotype flywheel system with a mean storage capacity of $2 \mathrm{~kW}-\mathrm{hr}$, by the end of the 1994 calendar year.

\section{The need for storage}

Commonwealth Edison (CECo) currently serves approximately 3.2 million customers covering the northem third of Illinois. A system capability 22,522 MW is currently in place with a load factor of approximately $57 \%$. CECo's generation capability mix is shown in Figure 1.

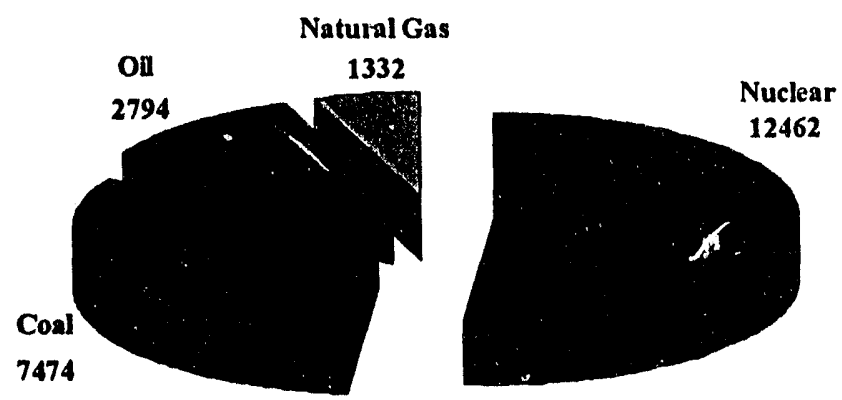

Figure 1 CECo Total Generation Capability (MWe)

Figure 2 illustrates the typical CECo system demand pattern. Note that the average day/night load swing covers some $4000 \mathrm{MW}$ of generation capacity. This forces severe load cycling of on-line units, frequent connecting and disconnecting generation from the grid, and limits the ability for inter-company transactions. System load analysis of the actual load curves indicates a need for approximately $10,000 \mathrm{MW}$-hrs of daily storage to be used/stored over a 5 hour period. Thus, 2,000 MW of storage based generation capability is desired on-line. With this storage capability, generation

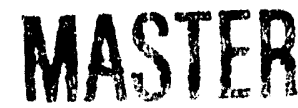

56th Annual American Power Conference
RECEIVED

\section{APR 181994}

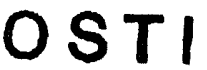


could remain fixed at approximately $8,500 \mathrm{MW}$. Charging would begin as load dips below 9,500 MW, while discharge (release to the grid) would commence as load exceeds this value.

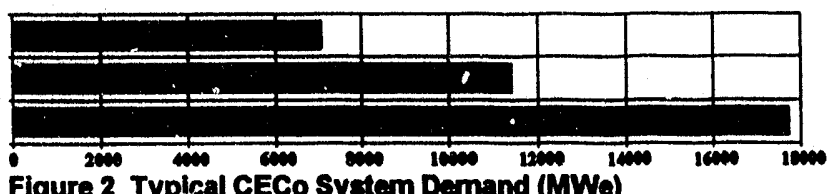

In addition to generation load swings, the transmission system also experiences these changes in load. A successful storage technology should take advantage of available night time transmission capacity to reduce the pressure on new line construction.

\section{Problems with current storage technologies}

Over the last 30 years, CECo has evaluated many different storage options. These include pumped hydro, compressed gas/air, batteries, and SMES. In the past, CECo has utilized the pumped hydro storage facility in Ludington, Michigan. However, due the relatively low overall system efficiency, excessive grid connection distance, and limited connection capacity, this option was not practical and it is no longer employed by CECo.

A number of potential storage technologies are currently available or under development for utility-scale energy storage. Table 1 outlines the benefits and disadvantages of each technology (shaded areas represent the most positive aspects).

From the table, it is seen that many technologies offer specific storage benefits. A successful device must have high storage density and total capacity, be modular, take advantage of existing transmission infrastructure, have low-cost operation, and a high duty cycle. HTS FES offers the potential, for the first time, to combine all of these features into a complete factoryconstructed package.

The base comparison is to gas turbine which is assumed to be used in the absence of any available storage option. In addition tu the drawbacks and benefits shown for gas turbines, it should be noted that the gas turbine option provides no improvement in existing capacity load factor and usually requires some transmission wire construction, as it typically cannot be placed in residential areas.

\begin{tabular}{|c|c|c|c|c|c|c|c|}
\hline & FES & $\begin{array}{l}\text { Bats- } \\
\text { eries }\end{array}$ & $\begin{array}{l}\text { Pumped } \\
\text { Hydro }\end{array}$ & $\begin{array}{l}\text { Comp- } \\
\text { ressed } \\
\text { Alruas }\end{array}$ & $\begin{array}{l}\text { Miero- } \\
\text { sMEs }\end{array}$ & SMEs ${ }^{a}$ & $\begin{array}{l}\text { Com- } \\
\text { bustion } \\
\text { Turbine }\end{array}$ \\
\hline Enciency & $3 x$ & $-70 \%$ & $-60 \%$ & $<50 \%$ & sory & $\infty$ & $-50 \%$ \\
\hline Enerry Capachi & $\sin$ & medium & 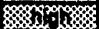 & $\log x$ & very low & s. & m \\
\hline Hodular & 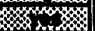 & sm & no & no & 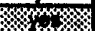 & no & $330 \mathrm{MW}$ \\
\hline CychLlfo & mons & $\times 100$ & $\times 1000$ & $\times 1000$ & 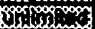 & $\operatorname{mos}$ & $\times 1000$ \\
\hline Charring Time & xymy & hours & hours & hours & Whoms & hours & 30 \\
\hline She Avallablitisy & $x+2$ & medium & Low & Jow & high & verylow & modium \\
\hline $\begin{array}{l}\text { Etored Energy } \\
\text { Measurement }\end{array}$ & 30 sym & poor & 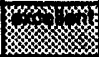 & $5 \times x$ & 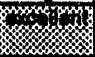 & $8 \times 48$ & $8 \%$ \\
\hline $\begin{array}{c}\text { Conetruction } \\
\text { Time }\end{array}$ & mas & months & yoars & yoars & 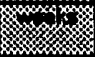 & years & monthe \\
\hline $\begin{array}{c}\text { Environ-mental } \\
\text { Impect }\end{array}$ & somes & large & vory large & very large & 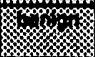 & very large & large \\
\hline $\begin{array}{c}\text { Acoldent } \\
\text { Conerequences }\end{array}$ & 5 & medium & high & medium & low & high & medium \\
\hline $\begin{array}{c}\text { Environmental } \\
\text { Controls }\end{array}$ & & $\begin{array}{l}\text { lonif- } \\
\text { leant }\end{array}$ & some & some & M & & some \\
\hline $\begin{array}{c}\text { Thermal } \\
\text { Requiroments }\end{array}$ & $\mathrm{LN}_{2}{ }^{\mathrm{b}}$ & olevated & 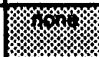 & high & c & $\mathrm{LHo}^{\mathrm{C}}$ & high \\
\hline Avallablity & $\begin{array}{l}\text { under } \\
\text { develop- } \\
\text { ment }\end{array}$ & & 禁 & \%ow & $\begin{array}{c}\text { under } \\
\text { develop- } \\
\text { ment }\end{array}$ & $\begin{array}{c}\text { under } \\
\text { develop- } \\
\text { ment }\end{array}$ & \\
\hline
\end{tabular}

Table 1 - Comparison of Energy Storage. Technologies

$a_{\text {SMES }}=$ Superconducting magnetic energy storage

$\mathrm{b}_{\mathrm{LN}_{2}}=$ Liquid nitrogen

$c_{\mathrm{LHe}}=$ Llquid helium

\section{Description of storage unit - how it works}

A flywheel energy storage system has essentially three main components: 1 . the bearing system for rotor support; 2 . the rotor system for energy storage and; 3. the motor/generator system (M/G) for energy conversion and power management. Figure 3 schematically illustrates these major components. Additionally, several other support functions are required, including vacuum, cryogenic, containment, and control systems.

The bearing is composed of two major components; a levitated rotating permanent magnet (PM), and hightemperature superconducting material (HTS) cooled to a minimum of $77 \mathrm{~K}$ (saturated liquid nitrogen). The principle of operation of the bearing is described in detail in section 6 .

The arrangement of Figure 3 shows two major cavities in the FES casing. The upper casing contains the rotor assembly and all spinning hardware. This cavity must be evacuated to at least $1.0 \times 10^{-5}$ torr to eliminate air friction losses. It also must provide structural support for the M/G stator assembly. The rotor, located in the upper cavity, is directly attached to the bearing magnet.

The lower casing houses the HTS material making up the other half of the bearing. This section of the FES operates at atmospheric pressure and is cooled to $77 \mathrm{~K}$.

The magnetic flux which links the permanent magnet 
and the HTS material is transmitted through the casing structure between the upper and lower cavities.

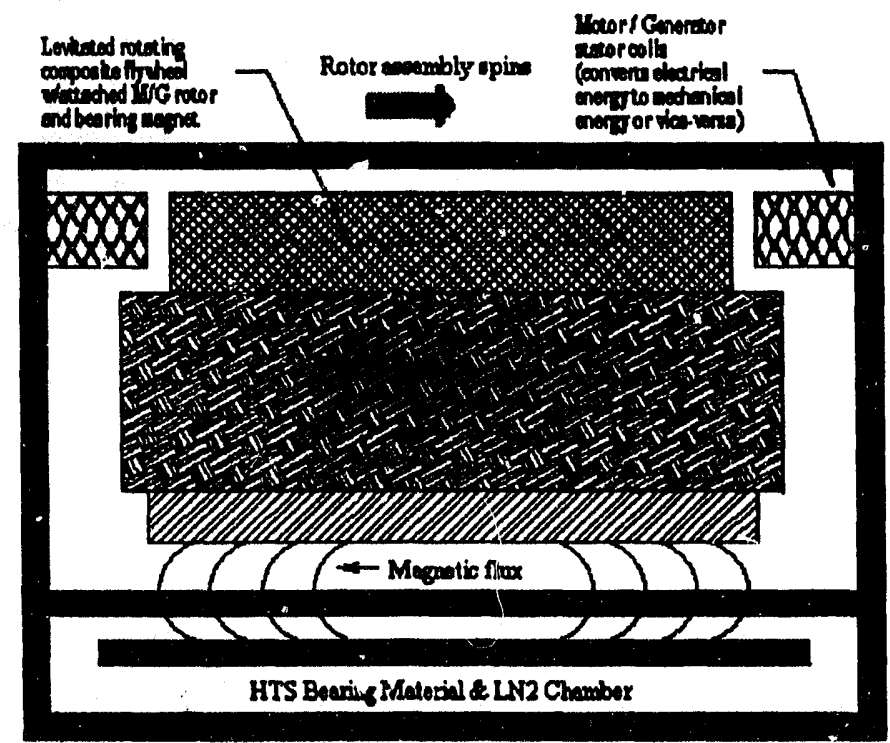

Figure 3. Schematic of FES

\section{The enabling technologies}

In the past, flywheel based concepts have been proposed for energy storage. However, basic limitations in materials and component performance have prevented the design of an efficient machine. Three major advancements in last few years have caused a reevaluation of the flywheel energy storage concept.

- High temperature superconducting bulk materials for ultra-low iriction passive magnetic bearings

- High strength composite fibers for constructing high speed flywheel rotors

- High performance power electronics allowing efficient energy conversion

Existing bearing technologies, including active nagnetic bearings, have two primary deficiencies. The frictional losses are so high that fly wheel stored energy is lost at rates greater than $1 \% /$ rour. This leads to $1 / 4$ of the stored energy lost per day. Secondly, the conventional bearings cannot manage the very high rotational speeds over the required long periods of operation.

The energy contained in a kernel of rotor mass is defined as:

$$
\text { K.E. }=1 / 2 m_{i} v_{i}^{2}
$$

where: $K . E .=$ stored kinetic energy of the rotor kernel $m_{1}=$ the mass of the rotor kernel $v_{1}=$ the tangential velocity of the rotor kernel

From the equation it can be seen the energy storage increases by the square of the velocity, while only linearly relative to mass. In order achieve reasonable storage energy densities, rotor tangential speeds must approach $1000 \mathrm{~m} / \mathrm{sec}$. This is the basis for the trend in modern flywheel systems towards higher speeds.

The physical rotor speed limit is governed by the relationship between the hoop stress experienced by the rotor and the rotor material strength. The rotor stress components are also strongly influenced by increasing mass. Additionally, the hoop stress is essentially independent of rotor radius and is a function of tip speed. Therefore, small rotors experience the same strength limitations as large rotors.

Current metal alloys are capable of only a few hundred $\mathrm{m} / \mathrm{sec}$ due to their relative low strength and high density. With the development of graphite fibers in low density composite matrices, material ultimate strengths of $1 \times 10^{6}$ psi are possible. These new materials can achieve rotor tip speeds potentially well in excess of $1000 \mathrm{~m} / \mathrm{sec}$. Additionally, composite materials offer certain advantages in their mode of failure, making containment of rotor failures a more manageable problem. Specifically, as a failure propagates throughout the rotor, a significant amount of energy is taken up in the destruction of the matrix chemical bonds. Metals, on the other hand, break into macroscopic pieces with little energy being expended in the propagation of the fractures.

Efficient power conversion, filtering and switch gear are critically important to overall operation of the FES. Conversion must be accomplished from $12 \mathrm{kV} 60 \mathrm{~Hz}$ to rotational mechanical energy and back to $12 \mathrm{kV}, 60 \mathrm{~Hz}$ with a minimum of $94 \%$ efficiency. The output wave form must approximate a true sinusoid function as induced noise and harmonics may be jetrimental to customer loads. Input/output power is nominally $1 \mathrm{MW}$. Losses are primarily concentrated in 3 areas:

- rectification,

- switching in inverter and motor drive commutation,

- and, filtering of the output wave form.

The combination of new high power switching components, such as new GTO's, IGBT's, advanced 
conversion circuitry, and transformers make this possible.

\section{Description of Bearing Technology}

The essential component of a high-efficiency flywheel is a low-loss superconducting bearing. Such bearings are typically composed of a permanent magnet levitated over a high-temperature superconductor (HTS). When a permanent magnet is brought close to a HTS, the HTS acts like a diamagnet. Superconducting shielding currents begin to circulate in the HTS in such a manner as to prevent magnetic flux from entering the interior of the HTS.

The configuration of magnetic field lines outside the HTS is essentially the same as if an "image magnet" were located the same distance below the surface of the HTS. The magnet floats over the HTS at a height at which the repulsive force between the real and image magnet is equal to the weight of the magnet. Technologically useful HTS contains extremely small nonsuperconducting regions called pinning centers. The superconducting bearing is passively stable in all directions because some of the magnetic flux lines penetrate into the HTS and become locked in place at these pinning centers.

HTS materials must be placed in a cold environment, such as in liquid nitrogen, to obtain the superconducting state. The best HTS material for levitation at liquid nitrogen temperature is co npused of $\mathrm{Y}-\mathrm{Ba}-\mathrm{Cu}-\mathrm{O}$. For high levitation pressure it $i_{s}$ important to have good flux pinning and also to have samples composed of large crystal grains, preferably $1 \mathrm{~cm}$ or larger in diameter.

Most superconducting bearing designs are symmetric about the rotation axis. The levitated permanent magnet is usually either a cylindrical disk or a cylindrical ring. The magnetic levitation pressure is proportional to the square of the magnetic field that the permanent magnet can exert on the HTS. The highest fields are obtained from FeBNd or SmCo, with internal magnetizations of $1.2 \mathrm{~T}$ commercially available. The permanent magnet is attached to the rest of the flywheel and must rotate in a vacuum. The HTS is typically contained in a cryogenic chamber which is flooded with liquid nitrogen.

The highest levitation pressures are obtained when the magnet is close to the HTS. Pressures greater than 100 $\mathrm{KPa}(15 \mathrm{psi})$ are readily attainable with simple bearing designs and present materials.

\section{Description of FES hook up to grid}

In order to achieve the maximum benefit of FES on both generation and transmission assets, FES devices must be sized for placement at the substation level as close to the load as possible. For CECo's system, the optimum size appears to be units with $5 \mathrm{MW}$-hr of capacity and $1 \mathrm{MW}$ of capability, attached at the $12 \mathrm{kV}$ voltage level. From Figure 2, approximately 10,000 MW-hrs of storage are needed to needed to compensate for daily load swings. This equates to 2,000 FES units dispersed throughout the CECo grid.

To manage such a large fleet, FES units will have to be designed for a minimum of human interaction. This includes the use of intelligent dispatching for autonomous control of voltage and VAR conditions found locally at the installation site, significantly reduce the demand on real time dispatching and operating man-power. The on-board control system, will also have to be capable of handling system faults and automatic breaker resets for long term unattended operation. Additionally, the FES fleet should operate independently to provide outage support during severe storms.

Several approaches are being considered for FES fleet dispatch. In a typical configuration, $80 \%$ of the storage fleet will be dispatched for 5 hour charge from 1200 to 0500; idle for 8 hours; and then release (generate) from 1300 through 1800 hours. The other $20 \%$ of the fleet will be dedicated to real time AGC (load follow), allowing virtually constant generation by the base load generating plants. Because the storage is placed at the load feeder, large industrial load swings will not propagate back through the grid, making AGC faster and more accurate.

Finally, high performance FES systems offer new opportunities for CECo to explore the use of alternative generation sources, such as wind and solar. One inherent problem with these sources has been their unpredictable production variability, further aggravating CECo's AGC difficulties. With an installed fleet of FES units handling real-time $A G C$, generation variability can be managed as well as load variability.

\section{Description of project and summary of results to date}

Commonwealth Research Corporation (CRC), Argonne National Laboratory and the US Department of Energy are engaged in a collaborative R\&D project to develop 
very low friction bearings for FES applications. The work has focused on utilizing the remarkable characteristics of bulk HTS materials for the development of passive magnetic bearings that do not require the elaborate feedback control systems needed by conventional active magnetic bearings. Initial HTS magnetic Learing investigations utilized a bell-jar vacuum chamber to measure the low friction coefficients attainable in the absence of air drag. Future development will focus on scale-up tests using the large vacuum test assembly described later.

CRC has provided overall project coordination, system engineering, and has evaluated utility interface requirements. The FES HTS magnetic bearing development team includes groups at Argonne involved both in the processing of HTS materials with improved levitation characteristics and in the design, development and testing of improved bearing technology. Consultants, manufacturers, and other organizations have been brought in to assist the project as the need arises. In particular, UGIMAG Inc. (Valparaiso, IN) has provided expertise in the fabrication of large NdFeB permanent magnets for rotor assemblies, Ability Engineering Technology (South Holland, IL) designed and fabricated a large FES vacuum test chamber, and Pennsylvania State University (Dept. of Engineering Science and Mechanics, University Park, PA) contributed to the development of graphite/epoxy composite rotor rims.

A number of spin-down tests have been conducted in a vacuum chamber consisting of a glass bell-jar chamber connected to an oil diffusion pump. The chamber is approximately $27 \mathrm{~cm}$ in diameter and contains a liquid nitrogen cryogenic chamber within the vacuum. HTS superconductors can either be placed inside the liquid nitrogen chamber or placed on top. The diffusion pump can create a vacuum of 10.7 Torr when the cryogenic chamber is cold. This apparatus has been used to measure spin-down of flywheels levitated with HTS bearings. The flywheels typically have less than a $1 \mathrm{~kg}$ mass and about a 10 to $15 \mathrm{~cm}$ diameter. The rotation rate is measured by a tachometer and the flywheel position determined by a traveling telescope. This apparatus has been used as a proving ground for techniques used in the larger vacuum chamber spindown apparatus. Spin-up has been accomplished both with gas-jets and with induction motors.

The figure of merit for the losses associated with a superconducting bearing is the coefficient of friction, $\mu$, defined as the drag force divided by the total levitated weight. Typically, a conventional roller bearing will have $\mu=0.001$. In our bell jar chamber we have obtained a coefficient of friction of $3 \times 10^{-7}$, which is believed to be a world's record for lowest friction in a superconducting bearing.

Recent project efforts have focused on development of test equipment to demonstrate scale-up of the HTS magnet bearing results obtained in the bell-jar test rig. For this purpose, a FES test apparatus (FTA) has been designed and constructed, and is currently undergoing shakedown tests. The main FTA test chamber is a cylindrical vessel constructed of stainless steel with an inside diameter of 36 inches and height of 34 inches. An LN2 cryochamber housing the HTS-bearing stator elements is housed within the FTA vacuum chamber. The FTA system includes a 1000 liter/sec turbomolecular vacuum pump and two rotary vacuum pumps to meet test plan requirements.

The key to the FTA design is versatility, in that it is adaptable to a variety of FES rotor and HTS magnetic bearing configurations and test cycle operating conditions. All FTA chamber viewports and instrument feed-through flanges use metal gasket seals for highvacuum service. The viewport windows are replaceable with blank-off flanges duing high-speed rotor tests as an added safety precaution. The top and bottom test chamber flanges include ports for: feed-through penetrations (LN2 lines, etc.), rotor positioning mechanisms, data acquisition cables, vacuum gages, and other instrumentation.

A motor/generator unit is being developed for integration with the FTA to demonstrate efficient FES power input/output. FES HTS magnetic bearing tests during 1994 will include rotors up to about 15 inches $O D$ and 25 pounds weight. These rotors will have an ultimate storage capability 2 - $5 \mathrm{~kW}$-hrs.

\section{Manufacturing and deployment}

In order for FES to be economically viable, storage units must be able to be produced for $\$ 500 / \mathrm{kW}(\$ 100 / \mathrm{kW}-\mathrm{hr}$ ) and provide energy for less than $\$ 0.05 / \mathrm{kW}$-hr. These pricing requirements are driven by the low cost generation alternative, single cycle gas turbine. In order to meet these very stringent cost requirements, three primary design characteristics must be achieved:

- standardization and simplicity of design, manufacture, and installation

- unit reliability/availability exceeding $99 \%$ with little 
or no operator interaction

- total 24 hour turn around energy efficiency exceeding $90 \%$

In order to accomplish low cost manufacture, selected manufacturers are being brought in very early in the design process to assist with simplifying the design for manufacture as well as provide prototype hardware. Design emphasis is being placed on advanced integrated materials rather than complex discrete engineered systems. Large scale, high volume manufacture of simple modular units is key to low cost FES.

FES units are being designed such that once a site is prepared, unit installation requires only a matter of hours. This lowers both the cost of initial installation, and provides for shop repair with a "pick-up and delivery" type of maintenance approach.

Because of the competitive market forces present in the utility industry today, the FES deployment schedule is exceptionally condensed. With the successful implementation of 2-5 $\mathrm{kW}$-hr prototype this year, a rapid development program progressing at an order-ofmagnitude per year pace is being pursued. Full field testing of a $1 \mathrm{MW}$-hr unit is planned for 1997, with production units of $5 \mathrm{MW}$-hr available later that year. This accelerated schedule demands a significant departure from normal utility design philosophy. Parallel component development, test-in-service, and other accelerated design approaches are being used and planned.

\section{Conclusion}

With the advent of high-temperature superconducting (HTS) materials, along with advancements in composite materials and power electronics, economical deployment of Flywheel Energy Storage (FES) may now be possible. Commonwealth Research Corporation (CRC), the research arm of Commonwealth Edison Company, and Argonne National Laboratory are implementing a demonstration project to advance the state of the art in HTS bearing performance and the overall demonstration of efficient Flywheel Energy Storage. HTS FES systems can reduce demand-based power plant cycling by storing unused nighttime capacity until it is needed to meet daytime demand.

To date, no physical limitations to rapid development of full sized FES systems have been discovered. Required bearing performance has been exceeded in laboratory scale tests and is now being prototyped in engineering scale systems along with rotor and power conversion technology. 


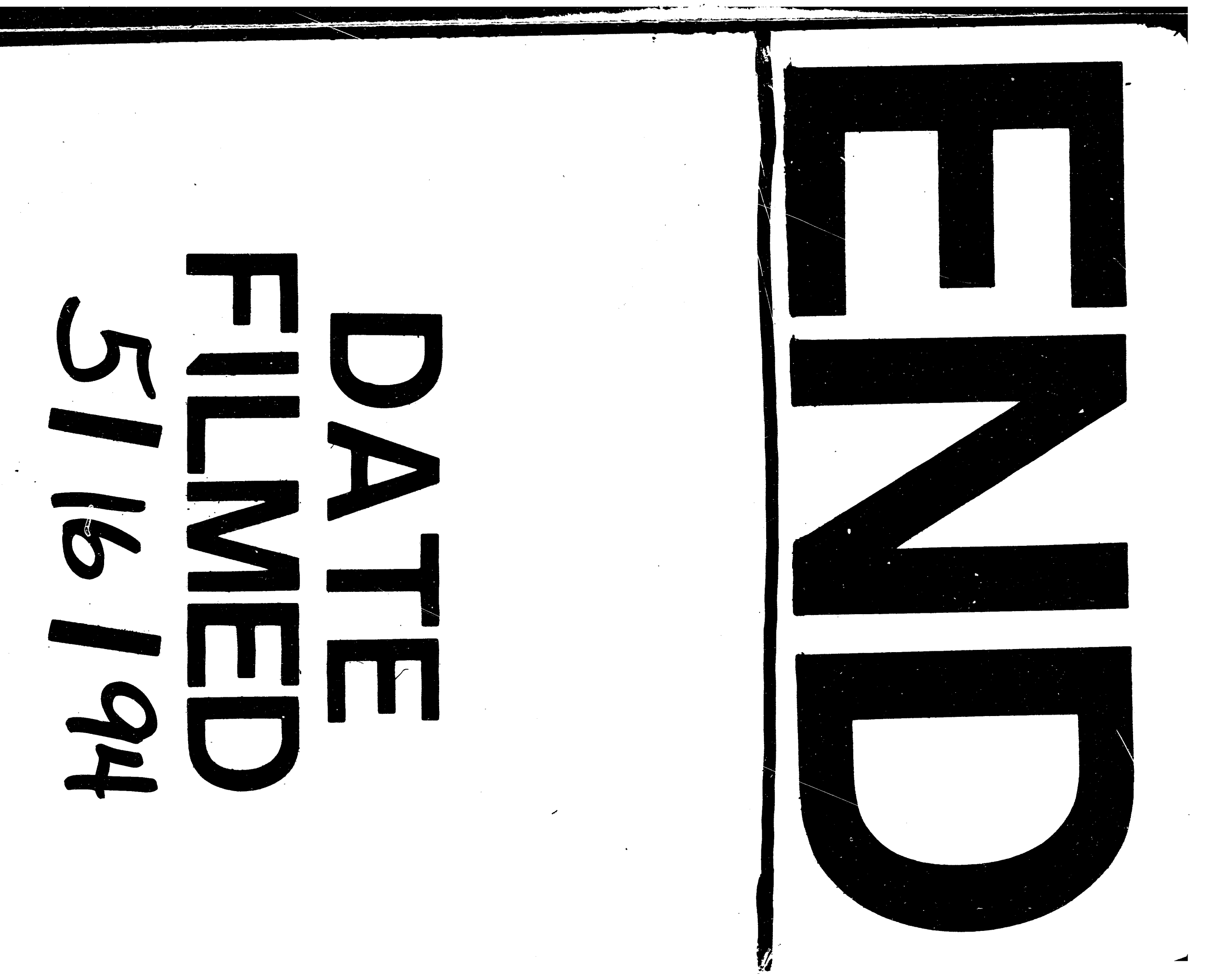


